\title{
Tetrahydrobiopterin-responsive hyperphenylalaninemia/phenylketonuria
}

INSERM

\section{Source}

INSERM. (1999). Orphanet: an online rare disease and orphan drug data base.

Tetrahydrobiopterin-responsive hyperphenylalaninemia/phenylketonuria. ORPHA:293284

Tetrahydrobiopterin-responsive hyperphenylalaninemia/ phenylketonuria ( $\mathrm{BH} 4-$

responsive hyperphenylalaninemia/ phenylketonuria) is a form of phenylketonuria (PKU, see this term), an inborn error of amino acid metabolism, characterized by mild to moderate symptoms of PKU including impaired cognitive function, seizures, and behavioral and developmental disorders, and a marked reduction and normalization of elevated phenylalanine concentrations after oral loading with tetrahydrobiopterin ( $\mathrm{BH} 4$; sapropterin dihydrochloride), an essential cofactor of phenylalanine hydroxylase. 